\title{
Elaboração de sobremesa láctea achocolatada com café
}

\author{
Mariana Saurin Tolfo, Bruna Lago Tagliapietra, Alvaro da Cruz Carpes, Ana Carolina \\ Mendes Dias Seibt, Neila Silvia Pereira dos Santos Richards
}

https://doi.org/10.4322/mp.978-65-991393-2-1.c3

\begin{abstract}
Resumo
O consumo de sobremesas lácteas tem apresentado importante crescimento nesta última década, justificado pelo progresso tecnológico em ingredientes e processos. Este estudo teve a finalidade de desenvolver sobremesa láctea achocolatada com café. Três formulações foram desenvolvidas, uma considerada padrão, sem adição de café, e duas com adição de café, com concentrações de 0,4 e 1,6\%. Foram realizadas análises físico-químicas para avaliação do pH e atividade de água, e determinação dos teores de umidade, cinzas, proteína, gordura e carboidratos. Testes afetivos de ordenação-preferência e intenção de compra foram aplicados em 50 avaliadores que consomem este tipo de produto. Os resultados mostraram que o produto desenvolvido é viável, oferecendo um produto com sabor mais acentuado. Os testes sensoriais aplicados indicaram que a sobremesa láctea com $0,4 \%$ de café foi escolhida a preferida pelos provadores e o teste de intenção de compra mostrou que $100 \%$ e $70 \%$ dos provadores comprariam a sobremesa láctea achocolatada com 0,4\% e com 1,6\% de café, respectivamente. Os resultados mostraram boa aceitação dos produtos por parte dos consumidores, e a adição de café não apresentou diferença estatística significativa quando avaliadas as propriedades físico-química das amostras analisadas.
\end{abstract}

Palavras-chave: Análise sensorial, cacau em pó, café, derivado lácteo, sobremesa láctea.

\section{Introdução}

Os produtos lácteos são consumidos em todo o mundo, e suas diferentes características nutricionais e sensoriais estimulam e favorecem seu consumo por diversos grupos da população. O mercado de sobremesas lácteas tem apresentado aumento nas últimas décadas, impulsionado pelos avanços tecnológicos das indústrias de laticínios, e dos ingredientes utilizados cada vez mais inovadores, o que possibilita a otimização de produtos com maior valor nutricional, variedade de texturas, sabores e aparências [1].

Em 2020 o MAPA (Ministério da Agricultura, Pecuária e Abastecimento) publicou a Instrução Normativa $n^{\circ} 72$ [2] que estabelece o Regulamento Técnico de Identidade e os Requisitos de Qualidade que devem apresentar as sobremesas lácteas. A sobremesa láctea é definida como o produto lácteo pronto para o consumo, composto pela mistura de leite, em suas diversas formas, padronizado ou não em seu teor de gordura, proteína, ou ambos, com derivados lácteos ou substancias alimentícias, ou 
ambos, podendo ser adicionada de amidos, amidos modificados e maltodextrina. As sobremesas lácteas deverão apesentar mais que $50 \% \mathrm{~m} / \mathrm{m}$ (cinquenta por cento massa/massa) de leite e outros produtos lácteos, isolado ou em combinação, do total de ingredientes do produto e, ainda não será permitida a adição de gordura vegetal em substituição à gordura láctea.

As sobremesas lácteas podem apresentar consistência semi-sólida, pastosa, sólida, aerada, gelificada, entre outras e são basicamente formuladas com leite, hidrocolóides, aroma e corante. Sua estabilidade depende da tecnologia de fabricação, características intrínsecas de cada ingrediente e estocagem sob condições refrigeradas [2, 3]. O processo de fabricação é constituído basicamente das etapas de preparo da mistura, tratamento térmico, homogeneização, resfriamento parcial e estocagem sob refrigeração [4].

Sobremesas lácteas achocolatadas são amplamente consumidas em todo o mundo, por consumidores de diferentes faixas etárias, sendo que os maiores consumidores são crianças até 10 anos de idade, responsáveis por quase $50 \%$ do volume consumido. Os lácteos achocolatados vêm despertando atenção na indústria alimentícia devido as suas características sensoriais como cor, sabor e aroma [5].

O desenvolvimento de novos produtos é um desafio para a indústria alimentícia, à medida que procura atender à demanda dos consumidores por alimentos saudáveis e atrativos [6]. Os ingredientes inovadores e sistemas tecnológicos aplicados nas indústrias de laticínios proporcionam novas alternativas às sobremesas clássicas, permitindo a produção de novos sabores, e produtos com maior digestibilidade e valor nutritivo [4]. Um dos ingredientes que pode ser utilizado na formulação deste produto é o café.

O café é um produto consumido diariamente no mundo por todas as classes sociais; desde o século XIX a importância da cafeicultura para o desenvolvimento econômico do Brasil é indiscutível [7]. Assim como as sobremesas lácteas, o café é um dos produtos mais consumidos no mundo. Segundo dados da Associação Brasileira da Indústria de Café (ABIC), o Brasil é o segundo maior consumidor de café do mundo, consumindo cerca de 1,07 milhões de toneladas no ano de 2017 [8].

Tendo em vista o grande consumo de café pelos brasileiros e o aumento da elaboração de sobremesas lácteas pela indústria de laticínios, o presente estudo teve por objetivo o desenvolvimento de um produto achocolatado com adição de diferentes concentrações de café. As amostras foram caracterizadas físico-química e sensorialmente, com 0 intuito de estabelecer o valor nutricional e a aceitação do produto.

\section{Materiais e Métodos}

A pesquisa foi desenvolvida no Departamento de Ciência e Tecnologia dos Alimentos na Universidade Federal de Santa Maria (UFSM), em Santa Maria (RS), Brasil.

Foram desenvolvidas três formulações de sobremesa láctea, uma sem adição de café, considerada formulação padrão e denominada SLO, e duas com concentrações diferentes de café, sendo denominadas SL1, a formulação com $0,4 \%$ de café e SL2 com $1,6 \%$ de café adicionado, de acordo com o estabelecido pelo Regulamento Técnico para Misturas para o Preparo de Alimentos Prontos para o Consumo (Resolução No 273, de 22 de setembro de 2005, da Agência Nacional de Vigilância Sanitária - ANVISA) [9]. Para assegurar a qualidade higiênico-sanitária do produto elaborado, foram atendidos os requisitos apresentados pela Portaria $n^{0} 326$ do 
Ministério da Saúde de 30 de junho de 1997, a qual dispõe de condições higiênicosanitárias e de Boas Práticas de Fabricação para estabelecimentos produtores/industrializadores de alimentos [10].

Para a preparação das sobremesas lácteas, foi utilizado leite integral UHT (Santa Clara $\AA$ ), goma xantana (Hexus Food $®$ ), açúcar (Alto Alegre $®$ ), sal (Diana $®$ ) e sabor chocolate (Selecta, Duas Rodas ${ }^{\circledR}$ ) nas mesmas proporções para as três formulações. De acordo com a formulações as quantidades de cacau em pó (Garoto®) e café solúvel em pó (Iguaçu®) foram ajustadas (Tabela 1).

\begin{tabular}{cccc}
\hline Ingredientes & SL0 & SL1 & SL2 \\
\hline Leite integral UHT & $2 \mathrm{~L}$ & $2 \mathrm{~L}$ & $2 \mathrm{~L}$ \\
(base) & $16(0,8 \%)$ & $16(0,8 \%)$ & $16(0,8 \%)$ \\
Goma xantana (g) & & & \\
Açúcar (g) & $240(12,0 \%)$ & $240(12,0 \%)$ & $240(12,0 \%)$ \\
Sal (g) & $4(0,2 \%)$ & $4(0,2 \%)$ & $4(0,2 \%)$ \\
Sabor chocolate (g) & $20(1,0 \%)$ & $20(1,0 \%)$ & $20(1,0 \%)$ \\
Cacau em pó (g) & $80(4,0 \%)$ & $72(3,6 \%)$ & $48(2,4 \%)$ \\
Café solúvel em pó (g) & - & $8(0,4 \%)$ & $32(1,6 \%)$ \\
\hline
\end{tabular}

Tabela 1. Formulações de sobremesa láctea achocolata com café.

$\mathrm{Na}$ elaboração das sobremesas lácteas, os ingredientes secos foram misturados e, posteriormente, adicionados de leite integral UHT. A foi pasteurizada a $85^{\circ} \mathrm{C}$ por 20 minutos, em agitação constante para a total dissolução e homogeneização dos ingredientes. Em seguida, as formulações receberam a adição do sabor chocolate e do café solúvel em pó, na concentração indicada (tabela1). Os produtos foram homogeneizados em mixer (11.000 rpm) durante 3 minutos, até completa dissolução.

O produto foi resfriado imediatamente, em banho de gelo, até temperatura de $10^{\circ} \mathrm{C} \mathrm{e}$ distribuído em embalagens plásticas assépticas, sendo armazenados em refrigerador à temperatura de $5 \pm 1{ }^{\circ} \mathrm{C}$ por 12 horas e, então, submetidos às análises físicoquímicas e sensoriais.

As análises físico-químicas foram: valor de pH (Potenciômetro Gehaka PG 1800) e atividade de água (Aqua-Lab - CX-2T calibrado com solução-padrão antes de cada aferição), determinação dos teores de umidade, cinzas, proteína (fator de correção 6,38 ) e carboidratos (diferença), de acordo com as recomendações do Instituto Adolfo Lutz [12]. A determinação de gordura seguiu o método de Bligh \& Dyer [11]. Todas as análises foram realizadas em triplicatas.

Foram realizados testes sensoriais afetivos de ordenação-preferência $\left(1^{\circ}, 2^{\circ}\right.$ e $\left.3^{\circ}\right)$ e de intenção de compra [12], aprovados pelo Comitê de Ética em Pesquisa com Seres Humanos da UFSM (CAAE 56769116.9.0000.5346). No teste de intenção de compra foi utilizada uma escala hedônica de cinco pontos (5 - certamente compraria, 4 provavelmente compraria, 3 - indiferente, 2 - provavelmente não compraria e 1 certamente não compraria) [13]. Os testes foram conduzidos em cabines individuais, com 50 provadores. As amostras foram servidas em copos descartáveis com $30 \mathrm{~mL}$ de amostra, codificados com números aleatórios de três dígitos. Para a limpeza do palato um copo com água mineral a temperatura ambiente foi disponibilizada aos provadores.

O valor energético da sobremesa láctea foi calculado de acordo com o recomendado pela RDC $n^{\circ} 360$, de 23 de dezembro de 2003, da ANVISA, utilizando os coeficientes de conversão de Atwater [14], ou seja, $4 \mathrm{kcal} / \mathrm{g}$ para proteínas, $4 \mathrm{kcal} / \mathrm{g}$ para 
carboidratos, $9 \mathrm{kcal} / \mathrm{g}$ para lipídios, e os resultados expressos em kcal por 100 gramas [15].

Os dados das análises físico-químicas foram analisados estatisticamente pela análise de variância (ANOVA) e a comparação das médias das amostras pelo Teste de Tukey ao nível de $5 \%$ de significância, o teste de ordenação-preferência pelo método analítico de Friedman [16] e o teste de intenção de compra através de análise de frequência (\%), a partir do programa estatístico SPSS ${ }^{2}$ versão 15.0.

\section{Resultado e Discussão}

Em produtos lácteos achocolatados, as gomas reagem com as proteínas do leite e as partículas do cacau a fim de formar uma rede tridimensional, que mantém as partículas em suspensão. Entretanto, para manter as partículas do pó de cacau em suspensão é preciso viscosidade relativamente alta [17].

Os resultados físico-químicos das sobremesas elaboradas estão apresentados na Tabela 2. Não houve diferença significativa entre os parâmetros físico químicos analisados. Os valores de pH das amostras variaram de 6,59 a 6,74. No estudo de Costa et al. [18], que avaliaram sobremesa láctea fermentada com diferentes tipos de estabilizantes, foi encontrado valores de $\mathrm{pH}$ em torno de 4,0; valores entre 5,77 e 7,15 foram encontrados no trabalho de Nikaedo et al. [19] com sobremesas lácteas achocolatadas cremosas utilizando concentrado proteico de soro, leite em pó integral e gomas carragena e guar.

A atividade de água ( $\mathrm{Aa}$ ) tem um papel muito importante na estabilidade do produto durante a sua vida de prateleira, conforme explica Silva et al. [20]. Valores de Aa de 0,2 a 0,3 podem baixar a velocidade de reação de oxidação dos ácidos graxos livres. Já a velocidade de oxidação pode aumentar quando os valores de atividade de água forem muito baixos (próximos de zero) e a valores muito elevados (próximos a um) [20]. O presente estudo encontrou valores de Aa em torno de 0,97 e Souza et al. [21], encontraram 0,93 de Aa em sobremesas lácteas de morango elaboradas a partir de soro de queijo.

O teor de proteína das sobremesas lácteas avaliadas, apresentou uma média de $4,32 \%$, corroborando com os teores encontrados por Souza et al. [21], que observaram teores de 4,5\% em amostras de sobremesa láctea sabor de morango elaboradas com soro de queijo. Naikaedo et al. [19] analisaram sobremesas lácteas achocolatadas cremosas e encontraram teores médios de 2,84\% de proteína, semelhante aos valores encontrados por Costa et al. [18], no estudo com sobremesa láctea fermentada, onde encontraram em torno de 2,41\%. Pode-se constatar que a sobremesa láctea achocolatada com adição de café possui um teor de proteína superior em relação à produtos desenvolvidos em estudos similares, e também ao preconizado pela IN 72 [2] que é de $2 \%$, o que atribui uma vantagem ao presente estudo.

Para valores de gordura, Soler et al. [22] encontraram valores médios de 5,8\% em sobremesa láctea achocolatada com adição de abacate, corroborando com 0 encontrado no estudo de Souza et al. [21], de 5,95\% de gordura, enquanto o presente trabalho encontrou valores médios de 1,72\%.

Quanto ao teor de carboidratos, Soler et al. [22] encontraram valores médios de 36,5\% nas sobremesas lácteas com adição de abacate, confirmando com os valores encontrados nas sobremesas lácteas achocolatada com café. 
As amostras avaliadas apresentaram teor médio de 1,16\% para cinzas, enquanto Soler et al. [22] encontraram média de 0,7\% em amostras de sobremesa láctea achocolatada com abacate. O teor de umidade das sobremesas lácteas achocolatadas com café foi de $62 \%$, semelhante ao teor encontrado por Souza et al. [21] de 67\%, e Soler et al. [22] que encontraram, em média, 55\%.

Todos os parâmetros analisados não apresentaram diferença estatística significativa entre os tratamentos SL0, SL1 e SL2, o que mostra que não houve interferência nas características físico-químicas dos produtos a adição de café, apenas interferências sensoriais, conforme mostram os resultados obtidos pelas análises físico-químicas (Tabela 2) e sensoriais (Tabelas 4 e 5).

\begin{tabular}{ccccc}
\hline Parâmetros & SL0 & SL1 & SL2 & CV (\%) \\
\hline $\mathrm{pH}$ & $6,59 \pm 0,14^{\mathrm{a}}$ & $6,63 \pm 0,28^{\mathrm{a}}$ & $6,74 \pm 0,98^{\mathrm{a}}$ & 1,09 \\
$\mathrm{Aa}$ & $0,96 \pm 0,00^{\mathrm{a}}$ & $0,97 \pm 0,00^{\mathrm{a}}$ & $0,97 \pm 0,00^{\mathrm{a}}$ & 0,05 \\
Proteína (\%) & $4,16 \pm 0,17^{\mathrm{a}}$ & $4,22 \pm 0,29^{\mathrm{a}}$ & $4,60 \pm 1,03^{\mathrm{a}}$ & 17,16 \\
Umidade (\%) & $62,83 \pm 0,08^{\mathrm{a}}$ & $62,75 \pm 0,00^{\mathrm{a}}$ & $62,73 \pm 0,24^{\mathrm{a}}$ & 2,39 \\
Gordura (\%) & $1,69 \pm 0,29^{\mathrm{a}}$ & $1,73 \pm 0,76^{\mathrm{a}}$ & $1,74 \pm 0,00^{\mathrm{a}}$ & 24,16 \\
Cinzas (\%) & $1,21 \pm 0,22^{\mathrm{a}}$ & $1,15 \pm 0,77^{\mathrm{a}}$ & $1,12 \pm 0,11^{\mathrm{a}}$ & 8,69 \\
Carboidratos (\%) & $30,11 \pm 1,98^{\mathrm{a}}$ & $30,79 \pm 2,43^{\mathrm{a}}$ & $30,18 \pm 1,40^{\mathrm{a}}$ & 6,50 \\
\hline
\end{tabular}

*Letras iguais na mesma linha não apresentam diferença significativa ao nível de $5 \%(p<0,05)$. Médias acompanhadas $\pm=$ Desvio Padrão. CV = coeficiente de variação. SLO $-4,0 \%$ de cacau em pó e 0\% de café; SL1 - 3,6\% de cacau em pó e 0,4\% de café; SL2 - 2,4\% de cacau em pó e $1,6 \%$ de café.

Tabela 2. Resultados das análises físico-químicas de sobremesas lácteas achocolatadas com café. Santa Maria, RS, Brasil.

A Tabela 3 refere-se ao valor energético de cada sobremesa com adição de café, mostrando que a porção de $100 \mathrm{~g}$ de produto, possui em torno de $150 \mathrm{Kcal}$, sendo em torno de $120 \mathrm{Kcal}$ composta por açúcares.

\begin{tabular}{cccc}
\hline Macronutriente & SL0 & SL1 & SL2 \\
\hline Proteína (Kcal) & 16,64 & 16,88 & 18,40 \\
Gordura (Kcal) & 15,21 & 15,57 & 15,66 \\
Carboidrato (Kcal) & 120,44 & 123,16 & 120,72 \\
Total (Kcal em 100g de produto) & 152,29 & 155,61 & 154,78 \\
\hline
\end{tabular}

Considerou-se valor calórico de $4 \mathrm{Kcal} / \mathrm{g}$ de proteína, $4 \mathrm{Kcal} / \mathrm{g}$ de carboidrato e $9 \mathrm{Kcal} / \mathrm{g}$ de gordura. SL0 - 4,0\% de cacau em pó e 0\% de café; SL1 - 3,6\% de cacau em pó e 0,4\% de café; SL2 - 2,4\% de cacau em pó e 1,6\% de café.

Tabela 3. Valor energético das sobremesas lácteas achocolatadas com café em $100 \mathrm{~g}$ do produto. Santa Maria, RS, Brasil.

A análise sensorial avaliada pelo teste de ordenação-preferência mostrou que a sobremesa láctea achocolatada com $0,4 \%$ de café (SL1) foi escolhida como preferida por $56 \%$ dos provadores, ou seja, 28 dos 50 provadores assinalaram a amostra SL1 em primeira posição como preferida, entre as três amostras. Entretanto, a amostra com 1,6\% (SL2) foi escolhida por apenas dois provadores como preferida entre as três sobremesas. A amostra padrão (SL0) apresentou 40\% de escolha como preferida, 0 que mostra que muito consumidores estão habituados com sabores convencionais e preferem produtos tradicionais quando comparados a outros diferenciados. Ainda, pode-se perceber que o teor de $0,4 \%$ de café foi preferido, mostrando que a nova formulação foi bem aceita mesmo em relação à uma formulação padrão e considerada tradicional. A sobremesa com $1,6 \%$ de café, de acordo com os comentários dos provadores, mascarou o gosto do cacau e do sabor chocolate, que ficaram pouco perceptíveis. A Tabela 4 mostra os resultados do teste de ordenação-preferência, 
observa-se que não existe diferença entre as amostras SL0 e SL1 e, também, entre as amostras SL1 e SL2.

\begin{tabular}{l|c|c|c|c}
\hline \multirow{2}{*}{ Amostras } & \multirow{2}{*}{ Total } & \multicolumn{3}{|c}{ Amostras } \\
\cline { 3 - 5 } & & SL0 & SL1 & SL2 \\
\cline { 3 - 5 } & & 40 & 28 & 6 \\
\hline SL0 & 40 & -- & $12^{\text {ns }}$ & $34^{*}$ \\
\hline SL1 & 28 & & -- & $22^{\text {ns }}$ \\
\hline SL2 & 6 & & & -- \\
\hline
\end{tabular}

${ }^{*}$ As amostras diferem entre si; $n s=$ as amostras não diferem entre si a $p \leq 0,05$

SL0 - 4,0\% de cacau em pó e $0 \%$ de café; SL1 - 3,6\% de cacau em pó e $0,4 \%$ de café; SL2 $2,4 \%$ de cacau em pó e $1,6 \%$ de café.

Tabela 4. Resultado do teste afetivo de ordenação-preferência das amostras de sobremesas lácteas achocolatadas com café. Santa Maria, RS, Brasil.

Os resultados obtidos no teste de intenção de compra composta de cinco pontos estão apresentados na Tabela 5. Constatou-se que a amostra padrão, sem adição de café (SL0) apresentou maior índice de "certamente compraria" por parte dos consumidores, o que confirma a tendência do consumidor em escolher produtos com sabor conhecido e de consumo rotineiro. Entretanto, a sobremesa láctea achocolatada com $0,4 \%$ de café (SL1), apresentou $100 \%$ de índice de compra, incluindo a escala para "certamente" e "provavelmente compraria". O teor adicionado de 1,6\% pode-se considerar que foi menos aceito pelos provadores, porém, mais de $70 \%$ dos provadores indicaram que comprariam o produto.

\begin{tabular}{cccc}
\hline Escala hedônica & SL0 & SL1 & SL2 \\
\hline Certamente compraria & $96 \%(n=48)$ & $92 \%(n=46)$ & $58 \%(n=29)$ \\
Provavelmente compraria & $4 \%(n=2)$ & $8 \%(n=4)$ & $18 \%(n=9)$ \\
Indiferente & - & - & $10 \%(n=5)$ \\
Provavelmente não compraria & - & - & $14 \%(n=7)$ \\
Certamente não compraria & - & - & - \\
\hline
\end{tabular}

SL0 - 4,0\% de cacau em pó e 0\% de café; SL1 - 3,6\% de cacau em pó e 0,4\% de café; SL2 $2,4 \%$ de cacau em pó e $1,6 \%$ de café.

Tabela 5. Teste de intenção de compra para as amostras de sobremesa láctea achocolatada com café. Santa Maria, RS, Brasil.

\section{Conclusão}

As sobremesas lácteas achocolatadas com café apresentaram uma boa resposta perante aos provadores, sendo que a amostra preferida foi a com menor teor de café adicionado (0,4\%). De acordo com os resultados obtidos conclui-se que os teores de café adicionados às sobremesas lácteas não afetaram os parâmetros físico-químicos avaliados, podendo inferir que a elaboração de sobremesas lácteas com café mostrase como uma alternativa viável para o uso desse produto tão apreciado pelo consumidor brasileiro.

\section{Referências}

[1] Szydlowska A., Kolozyn-Krajewska D. Development of potentially probiotic and synbiotic pumpkin frozen desserts. CYTA- Journal of Food 2019, 17(1):251-259. https://doi.org/10.1080/19476337.2019.1570975.

[2] Brasil. Instrução Normativa no 72, de 24 de julho de 2020. Ministério da Agricultura, Pecuária e Abastecimento. Disponível em: https://www.in.gov.br/web/dou/-/instrucaonormativa-n-72-de-24-de-julho-de-2020-269156546. Acesso em 03/08/2020. 
[3] Ares F., Arrarte E., Léon T., Ares G., Gámbaro A. Development of functional milk desserts enriched with resistant starch based on consumers perception. Food Science and Technology International 2013; 18(5):465-475. https://doi.org/10.1177/1082013211433070.

[4] Silva A.S., Barros A.C.F., Oliveira E.T. Avaliação de parâmetros sensoriais em sobremesas lácteas sabor chocolate elaboradas com leite e soro de leite. VII CONNEPI 2012; Palmas, Tocantins. ISBN 978-85-62830-10-5.

[5] Eduardo M.F., Lannes S.C.S. Achocolatados: análise química. Revista Brasileira de Ciências Farmacêuticas 2004; 40(3):405-412. https://doi.org/10.1590/S151693322004000300017.

[6] Juliano R.S., Sarkis S.S.J., Pinheiro A.C., Fear A.C., Zambelli C.A., Augusto M.M. Desenvolvimento de Sobremesa Láctea Tipo Frozen Yogurt Com Características Funcionais. In: XX Congresso Brasileiro de Engenharia Química. 2014. Florianópolis. https://doi.org/10.5151/chemeng-cobeq2014-0412-25631-159563.

[7] Monteiro M.A., Minim V.P.R., Silva A.F., Chaves J.B.P., Cardello H.M.A.B. Perfil sensorial da bebida café (Coffea arábica) determinado por análise Tempo-Intensidade. Ciência e Tecnologia de Alimentos 2005; 25(4):772-780. https://doi.org/10.1590/S0101-20612005000400024.

[8] ABIC. Associação Brasileira da Indústria do Café. Consumo interno de café recupera crescimento e aumenta 1,24\%. Disponível em: http://www.abic.com.br/publique/cgi/cgilua.exe/sys/start.htm?sid=61\#1910. Acesso em 21 maio 2018.

[9] Ministério da Saúde. Agência Nacional de Vigilância Sanitária. Resolução no 273 , de 22 de Setembro de 2005. Aprova o Regulamento Técnico para Misturas para o Preparo de Alimentos e Alimentos Prontos para o Consumo. Brasília, Brasil, 2005.

[10] Brasil. Portaria $n^{\circ} 326$, de 30 de junho de 1997. Ministério da Saúde. Dispõe de condições higiênico-sanitárias e de Boas Práticas de Fabricação para estabelecimentos produtores industrializadores de alimentos. 1997.

[11] Bligh E.G., Dyer W.J. A rapid method of total lipid extraction and purification. Canadian Journal of Biochemistry and Physiology 1959; 37(8):911-7. https://doi.org/10.1139/059-099.

[12] IAL. Instituto Adolfo Lutz. Métodos físico químicos para análise de alimentos - $4^{\mathrm{a}}$ ed. Série Normas e Manuais Técnicos, Ministério da Saúde, ANVISA: Brasília, 2008,1020 p.

[13] Ferreira V.L.P., Almeida T.C.A., Pettinelli M.L.C.V., Silva M.A.A.P., Chaves J.B.P., Barbosa E.M.M. Análise sensorial: testes discriminativos e afetivos. Manual: série qualidade 2000. Campinas: SBCTA, 127p.

[14] Atwater W.O., Bryant A.P. 12th Annual Report (1899) of the Storrs, CT, Agricultural Experimental Station. Storrs, CT: Storrs Experimental Station 1900, p. 73110. 
[15] Agência Nacional de Vigilância Sanitária. Resolução RDC n. 360, de 23 de dezembro de 2003. Aprova o regulamento técnico sobre rotulagem nutricional de alimentos embalados. Brasília, Brasil, 2003.

[16] Palermo J.R. Análise sensorial: fundamentos e métodos. Rio de Janeiro: Editora Atheneu, 2015. 158p.

[17] Van Oorschot $N$. Perfeição em matéria de sobremesas e leites achocolatados. Leite e Derivados 2001; 10(58):28-31.

[18] Costa A.V.S., Nicolau E.S., Torres M.C.L., Fernandes P.R., Rosa S.I.R., Nascimento R.C. Development and physical-chemical, microbiological and sensory characterization of fermented dairy beverage prepared with diferente stabilizers/thickener. Semina: Ciências Agrárias 2013; 34(1):209-226. http://dx.doi.org/10.5433/1679-0359.2013v34n1p209.

[19] Nikaedo P.H.L., Amaral F.F., Penna A.L.B. Caracterização tecnológica de sobremesas lácteas achocolatadas cremosas elaboradas com concentrado protéico de soro e misturas de gomas carragena e guar. Revista Brasileira de Ciências Farmacêuticas 2004; $\quad$ 40(3):397-404. https://doi.org/10.1590/S151693322004000300016.

[20] Silva F.A.M., Borges M.F.M., Ferreira M.A. Métodos para avaliação do grau de oxidação lipídica e da capacidade antioxidante. Química nova 1999; 22(1):94-103. http://dx.doi.org/10.1590/S0100-40421999000100016.

[21] Souza J., Souza J., Souza C.F.V. Desenvolvimento, parâmetros físico-químicos e avaliação sensorial de sobremesas lácteas elaboradas com soro de queijo e gomas. Revista do Instituto de Laticínios Cândido Tostes 2013; 68(393):16-25. https://doi.org/10.5935/2238-6416.20130031.

[22] Soler N., Batista A.G., Faria C.A.M., Gonzaga D.G., Lopes J.M.M., Pinto N.A.V.D. Elaboração, composição química e avaliação sensorial de sobremesas lácteas achocolatadas com abacate. Alimentos e Nutrição 2011; 22(1):143-148.

\section{Autores}

Mariana Saurin Tolfo, Bruna Lago Tagliapietra, Alvaro da Cruz Carpes, Ana Carolina Mendes Dias Seibt, Neila Silvia Pereira dos Santos Richards*

Departamento de Tecnologia e Ciência dos Alimentos, Universidade Federal de Santa Maria - DTCA/UFSM, Avenida Roraíma, 1000, Prédio 42, sala 3211, 97105-900, Santa Maria - RS, Brasil.

\footnotetext{
* Autor para correspondência: neilarichardsprof@gmail.com
} 